\title{
An Effective Path Selection Method in Multiple Care-of Addresses MIPv6 with Parallel Delay Measurement Technique ${ }^{\star}$
}

\author{
Jungwook Song, Heemin Kim, and Sunyoung Han ${ }^{\star \star}$ \\ Department of Computer Science and Engineering, Konkuk University \\ 1 Hwanyang, Gwangjin, Seoul 143-701, Korea \\ \{swoogi, procan, syhan\}@konkuk.ac.kr
}

\begin{abstract}
In the Ubiquitous Society, there will be many types of mobile access network surrounding us and we can access the Internet anytime anywhere. At that time, mobile device can select several links from surrounded mobile access networks and access the Internet with multiple interfaces. We have already Mobile IPv6 protocol that supports mobility and try to extend to support multiple Care-of Addresses registration. But, we don't have any solution for selecting effective path. The effective path has many advantages such as reducing communication overhead. In this paper, we propose that effective path selection method in Multiple Care-of Addresses Mobile IPv6 environment with 'Parallel Delay Measurement' technique. With our technique, we can make down average packet delay.
\end{abstract}

\section{Introduction}

In the Ubiquitous Society, there will be many types of mobile access network surrounding us and we can access the Internet anytime anywhere. Nowadays, we can see that many types of devices keep contact to the Internet while moving their location. And there are many types of mobile access network services such as HSDPA(High Speed Downlink Packet Access), IEEE 802.16e mobile WiMAX(WIBRO in KOREA), and IEEE 802.11 Hotspots. HSDPA and mobile WiMAX are supporting high speed movement of mobile devices and IEEE 802.11 Hotspots support connection to the Internet in many public areas.

The network layer protocol is very important as the mobile access network. We have already standardized mobility support protocol that is Mobile IPv6[1]. According to the current Mobile IPv6 specification, a mobile node may have several care-of addresses, but only one, termed the primary Care-of Address, can be registered with its Home Agent and the Correspondent Nodes. However, for matters of cost, bandwidth, delay, etc., it is useful for the mobile node to get Internet access through multiple access media simultaneously, in which case multiple active IPv6 Care-of Addresses would be assigned to the mobile node. So,

\footnotetext{
* This research was supported by the 'Seoul R\&D Program'.

${ }^{\star \star}$ Corresponding author. 
IETF(Internet Engineering Task Force) Monami6 Working Group try to extend exists Mobile IPv6 protocol standard to support multiple Care-of Addresses registration $[2]$.

Many type of Adapters for mobile access network will become more cheaper and smaller. So, the Mobile devices can access multiple link to the Internet with multiple adapters and register multiple Care-of Addresses with MCoAMIPv6(Multiple Care-of Addresses Mobile IPv6). Not the less, there is no proper solution for selecting effective path(link) to the Home Agent or the Correspondent Nodes. There are many disadvantages cause of non-effective path.

In this paper, we propose the solution for selecting effective path in MCoAMIPv6 using 'Parallel Delay Measurement' technique. With our method, the Mobile Node and its Home Agent and the Correspondent Nodes can select effective path and we can reduce the communicating overhead.

The rest of the paper is organized as follows. Section 2 describes the path selection problem of MCoA-MIPv6. Section 3 describes the 'Parallel Delay Measurement' technique. Section 4 shows the result of analysis and concluding remarks are in Section 5.

\section{Path Selection Problem of MCoA-MIPv6}

\subsection{Current Problem}

The Mobile Node can have multiple interfaces and many types of mobile access network can be overlapped. So, it is needed that multiple Care-of Addresses registration for effectiveness and redundancy. Fig. 1 shows two sample configurations in the middle of overlapped mobile access networks.

Let's assume the CoA1(mobile access network associated with the Care-of Address1) has biggest bandwidth, minimum cost and minimum delay(different from one-way delay or round trip time to the Home Agent or to the Correspondent Nodes), it is natural that MN(Mobile Node) chooses CoA1 as the prime link to access the Internet, and it is reasonable in case (a) of Fig. 1. But in case
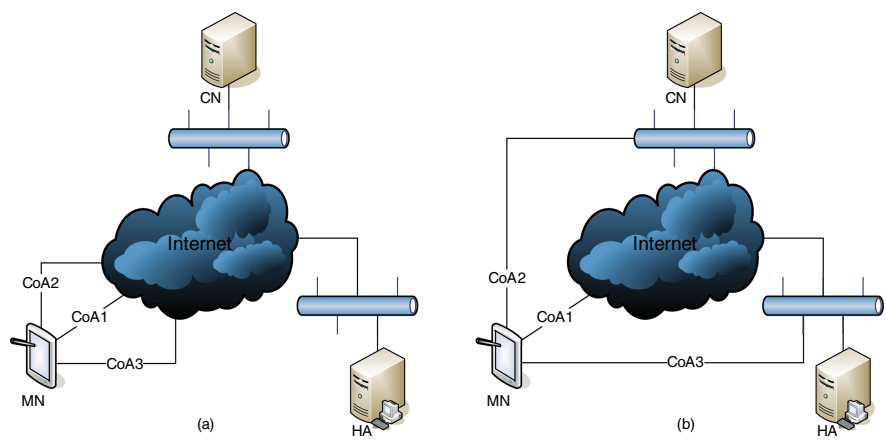

Fig. 1. Sample Configuration 
(b) of Fig. 1, we can easily guess that CoA2 is better than CoA1 when MN communicating with $\mathrm{CN}$ (Correspondent Node) and CoA3 is better than CoA1 when MN communicating with HA(Home Agent).

There are no functions that can measure whole path quality of communication parties in the MCoA-MIPv6, and they are actually needed. The Mobile Node just grades links and sets priority values to the Binding Unique Identifier suboption and the Correspondent Node refers to this priority values for selecting effective path of communication. That priority values represent only link specific characters, but communicating parties actually need path specific characters.

\subsection{Current Implementations of MCoA}

The SHISA is an implementation of Mobile IPv6 which supports MCoA. The WIDE project had developed two different Mobile IPv6 through the KAME project (KAMEMIP) and through the internetCAR project(SFCMIP). They finally decided to work together for single implementation (called SHISA) in the WIDE project on spring 2004. The SHISA support RFC3775, RFC3776, RFC3963, RFC4584, draft-wakikawa-mobileip-multiplecoa-05 and draft-momose-mip6-mipsock-00 345.

The NEPL NEMO Platform for Linux is the NEMO(NEtwrok MObility) implementation based on the MIPL2 architecture. This is another implementation of supporting $\mathrm{MCoA} 67$.

\section{Parallel Delay Measurement Technique}

In this section, we describe the 'Parallel Delay Measurement' technique for effective path selection in MCoA-MIPv6. We can measure one-way delay with addition of the Binding Timestamp sub-option to the MCoA-MIPv6 2]. From this one-way delay and exist priority values of the Binding Unique Identification sub-option, we can select effective path and reduce communicating overhead. And we also can be adapted to variation of link status through binding refreshment mechanism of MIPv6. Some modification to MCoA-MIPv6 are required to support parallel delay measurement technique.

\subsection{Binding Timestamp Sub-option}

To measure one-way delay, the Mobile Node must add timestamp information to its own Binding Update message. If needed, the Binding Timestamp sub-option is included in the Binding Update, Binding Acknowledgement, and Binding Refresh Request messages. Fig. 2 shows the format of the Binding Timestamp sub-option that we newly define in this paper. When the Mobile Node sends Binding Update message with the Binding Unique Identifier sub-option and the Binding Timestamp sub-option, the Priority/Status field in the Binding Unique Identifier sub-option can be set to specific value from the MCoAMIPv6 or set to zero for entrusting selecting path to the Correspondent Node. 


\begin{tabular}{|l|l|l|}
\multicolumn{1}{c|}{8} & 16 & 24 \\
\cline { 2 - 3 } \multicolumn{1}{c|}{} & \multicolumn{1}{c|}{ Type $=$ TBD } & Length \\
\hline Binding Unique ID (BID) & \multicolumn{2}{|c|}{ Reserved } \\
\hline \multicolumn{3}{|c|}{ Timestamp } \\
\hline
\end{tabular}

Fig. 2. Binding Timestamp sub-option

- Type

Type value for Binding Timestamp sub-option will be assigned later.

- Length

8-bit unsigned integer. Length of the option, in octets, excluding the Type and Length fields. This field must be set to 4 .

- Binding Unique ID (BID)

The BID which is assigned to the binding carried in the Binding Update with this this sub-option. BID is 16-bit unsigned integer. A value of zero is reserved 2 .

- Reserved

8 bits reserved field. Reserved field must be set with all 0 .

- Timestamp

32-bit unsigned integer. This value can be retrieved from normal C library function.

\subsection{Parallel Binding Update}

In MCoA-MIPv6, the Mobile Node has multiple interfaces and has multiple links to the Internet. So, the Mobile Node has multiple Care-of Addresses and sends the Binding Update messages to the Home Agent and to the Correspondent Nodes per each care-of address [2. In our method, when the Mobile Node sends the Binding Update messages, it sends all binding information of all Care-of Addresses simultaneously as possible. The Timestamp field in the Binding Timestamp suboption must be set as the value of the Binding Update message creation time. So, all Timestamp values are slightly different. When the Mobile Node receives the Binding Refresh Request message, it must send all binding information of each Care-of Address at the same time with Binding Timestamp sub-option.

And, when sending Binding Update messages, the Mobile Node sets all TTL(Time To Live) value of the IPv6 header belong the Binding Update messages as the same value. From this small technique, we can easily measure the difference of the hop count of each path.

\subsection{Selecting Effective Path on the Home Agent and the Correspondent Node}

In MCoA-MIPv6, the Mobile Node determines priorities of each Care-of Address and sends that to the Home Agent and the Correspondent Nodes with the Binding Unique Identifier sub-option [2]. In our method, priorities are synthetically 
determined by the Mobile Node, the Home Agent and Correspondent Nodes. Whenever the Home Agent receives the Binding Update message with Binding Unique Identifier sub-option and Binding Timestamp sub-option, gets its own timestamp at that time and calculates difference between the Binding Timestamp and its own timestamp. This difference value is the one-way delay. The Home Agent could get minimum one-way delay and gives higher priority value to that binding information. It is not need global timestamp or absolute one-way delay, we need just relative one-way delay. We could assume that relatively small one-way delay means small packet delay. The Correspondent Nodes determine priorities same as the Home Agent.

Because the Mobile Node sets all TTL values as the same value, we can easily measure the difference of the hop count of each path. When the Home Agent receives Binding Update messages, retrieves final TTL values from messages and decides maximum and minimum final TTL value. The smaller final TTL value means the bigger hop count and the bigger final TTL value means the smaller hop count. Thus, the maximum final TTL value means the shortest path.

From these two factor, one-way delay and final TTL value, we could calculate new priority value $P_{N E W}$ as following equation.

$$
P_{N E W}=\alpha P_{M N}+\beta \Delta T T L-\gamma \text { Delay }
$$

$P_{M N}$ means the priority value from the Mobile Node, $\triangle T T L$ means difference between final TTL value and minimum final TTL value and Delay means the one-way delay value. $\alpha, \beta$ and $\gamma$ are positive weight values that will be determined with real experiment.

\subsection{Selecting Effective Path on the Mobile Node}

When the Home Agent and Correspondent Node receive the Binding Update message and if that is correct, they send the Binding Acknowledgement

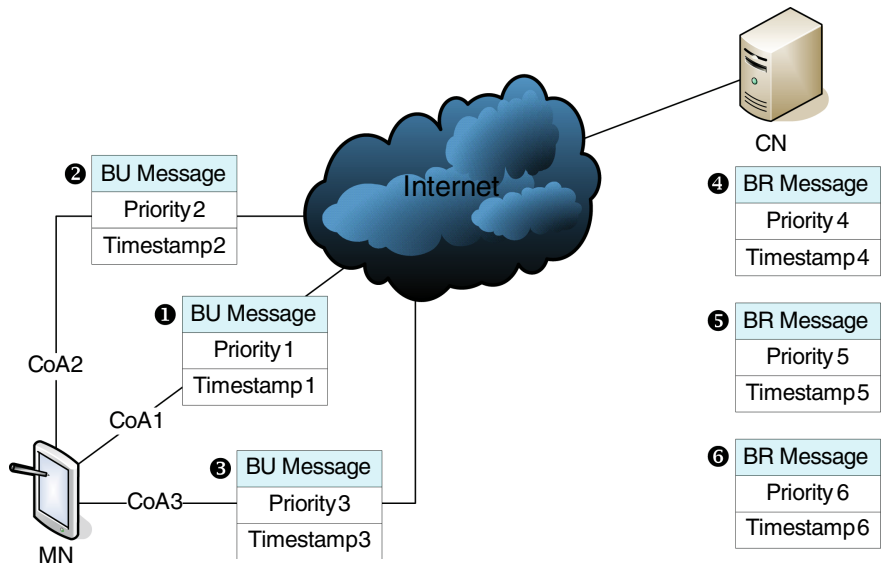

Fig. 3. Parallel Delay Measurement 
message[2]. At that time, in our method, they send the Binding Acknowledgement messages with Binding Timestamp sub-option. Then the Mobile Node receives the Binding Acknowledgement messages, calculates one-way delay and determines priorities.

Fig. 3 shows brief sequence of the parallel delay measurement technique.

(1) $\sim 3 \mathrm{MN}$ sends the BU messages to $\mathrm{CN}$ with priorities and Timestamps.

(4) $\sim 6$ CN replies BR messages to MN priorities and Timestamps.

\section{Analysis}

To analyze effectiveness of our method, we consider two cases of one scenario. The scenario is that the Mobile Node has three network connection and exchange one thousand messages with the Correspondent Node through the highest priority path. We take the 'tic-toc' model as exchanging messages, the Mobile Node sends a 'tic' message and the Correspondent Node receives 'tic' and reply with a 'toc' message. This is one sequence and repeat five hundred times. Totally they exchange one thousand messages.

The first case of the scenario, the Correspondent Node is plain IPv6 node, therefore the Mobile Node and the Correspondent Node can't optimize the routing path of packets. The second case of the scenario, the Correspondent Node is MIPv6 enabled node, therefore the Mobile Node and the Correspondent Node can optimize the routing path of packets.

And, we consider two network configurations as shown in Fig. 1. We assume that the average delays of each link as follows: CoA $\rightarrow 2 m s$, CoA2 $\rightarrow 3 m s$, $\mathrm{CoA} 3 \rightarrow 5 \mathrm{~ms}$ and internet cloud $\rightarrow 10 \mathrm{~ms}$. We don't need to consider the other

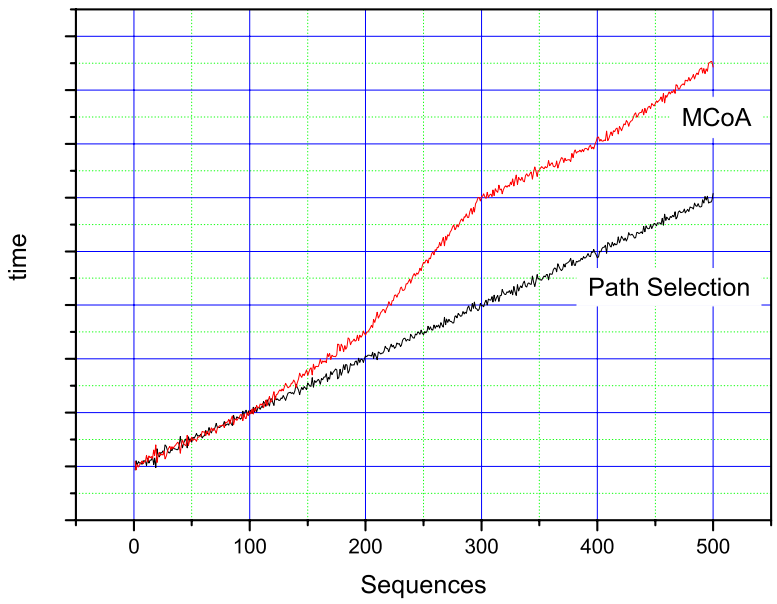

Fig. 4. Analysis Result of Configuration (a). Because Path Selection Method always could select better path, its performance is better. 


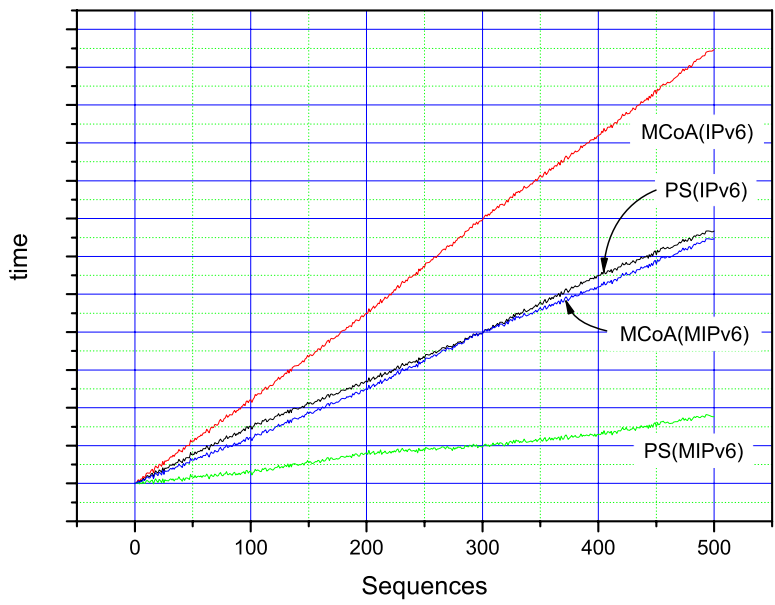

Fig. 5. Analysis Result of Configuration (b). Even if the Correspondent Node is not MIPv6 enabled, the path selection(PS) method is better.

link, because other link will any affect to the result. The delays of each link can be changed suddenly while exchanging messages, because the Mobile Node can moving. We assume that delays of each link rotate when after exchanging every 100th message. Fig. 4 and Fig. 5 show the results.

\section{Concluding Remarks}

In this paper, we proposed an effective path selection method in MCoA-MIPv6 with 'Parallel Delay Measurement' technique. To measure one-way delay, we defined new sub-option called the Binding Timestamp sub-option and through one-way delay, we could maximize the efficiency of communication between the Mobile Node and the Home Agent or the Correspondent Nodes.

We are building NGN(Next Generation Network) for the Ubiquitous Computing environment. The NGN has huge bandwidth compared with the present Internet. But, if hundreds of or thousands of application services for Ubiquitous Network are deployed, it will become crowded same as the present situation. So, we need the solution that maximize the utilization of the network. We believe that our method can contribute to increasing effectiveness of the network usage. We need more researches on context adaptive measurement method.

\section{References}

1. Johnson, D., Perkins, C., Arkko, J.: Mobility Support in IPv6: RFC3775, IETF (June 2004)

2. Wakikawa, R., Ernst, T., Nagami, K.: Multiple Care-of Addresses Registration: draft-ietf-monami6-multiplecoa-01.txt, IETF Draft (October 2006) 
3. SHISA, http://www.mobileip.jp/

4. The KAME project, http://www.kame.net/

5. WIDE Project, http://www.wide.ad.jp/

6. Nautilus, http://software.nautilus6.org/

7. MIPL, NEPL, http://www.mobile-ipv6.org/

8. Devrapalli, V., Wakikawa, R., Petrescu, A., Thubert, P.: Network Mobility (NEMO) Basic Support Protocol: RFC3963, IETF (January 2005)

9. Ng, C., Thubert, P., Watari, M., Zhao, F.: Network Mobility Route Optimization Problem Statement: draft-ietf-nemo-ro-problem-statement-03.txt, IETF Draft (September 2006)

10. Deering, S., Hinden, R.: Internet Protocol, Version 6 (IPv6) Specification: RFC2460, IETF (December 1998) 